\title{
High Precision Astrometry with Closure Constraints
}

E. Ros, J. M. Marcaide, \& J. C. Guirado

Dep. d'Astronomia i Astrofísica, U. de València, E-46100 Burjassot, Spain

M. I. Ratner, \& I. I. Shapiro

Harvard-Smithsonian Center for Astrophysics, Cambridge, MA 02138, USA

T. P. Krichbaum, \& A. Witzel

Max-Planck-Institut für Radioastronomie, 53121 Bonn, Germany

R. A. Preston

Jet Propulsion Laboratory, Caltech, 4800 Oak Grove, Pasadena, CA 91109, USA

Abstract. We determine the relative separations of the sources in the triangle $1803+784 / 1928+738 / 2007+777$ with submilliarcsecond accuracy from global $8.4 \mathrm{GHz} \mathrm{VLBI}$ observations. We remove the ionospheric contribution to the phase-delay observable using ionospheric total electron content estimates obtained from Global Positioning System (GPS) data. The triangular geometry provides a consistency check through sky closure.

\section{Introduction}

The use of differenced fringe phases from VLBI observations of two sources separated by a few degrees yields relative positions of radio sources with submilliarcsecond accuracies and with fractional precisions $\sim 2 \cdot 10^{-9}$ (cf. Guirado et al. 1995a,b; Lara et al. 1996). In this paper, we report on results obtained using a new scheme: observation of triangles of radio sources, which provide new geometrical constraints. In radio astrometric work, the ionospheric contribution is an important one. Sardón, Rius, \& Zarraoa (1994) have shown that the total electron content (hereafter TEC) of the ionosphere for any line of sight can be determined with high accuracy by using dual frequency (1575.42 MHz and $1227.60 \mathrm{MHz}$ ) GPS data. We use this approach to sharply reduce the ionospheric effect in our phase-delay data.

\section{VLBI Observations and Astrometric Data Reduction}

We observed the radio sources $1803+784,1928+738$, and $2007+777$ (selected from the S5 sample, Witzel et al. 1988) cyclically every 9 minutes, simultaneously at 2.3 and $8.4 \mathrm{GHz}$ on November $20-211991$ for $14 \mathrm{hr}$ with a global VLBI array, using MkIII instrumentation. Due to technical problems we were able to utilize data only from the following subarray of the original array: Effelsberg, Fort Davis, Pie Town, Kitt Peak, and Los Alamos. The data were correlated at the MPIfR (Bonn, Germany).

Following, e.g., Shapiro et al. (1979), we construct "phase-connected" delays. We then form the differenced phase-delay observable by subtracting from the value of the phase-delay from the observation of each source the corresponding value from the observation of the following source. We use both the undifferenced and differenced phase-delays at $8.4 \mathrm{GHz}$ to estimate the angular separations between the sources via a weighted-least-squares fit. In our model, we correct for the effects of the tropospheric delay (site dependent) and the structure of the radio sources, and allow for possible overall phase-delay ambiguities. 


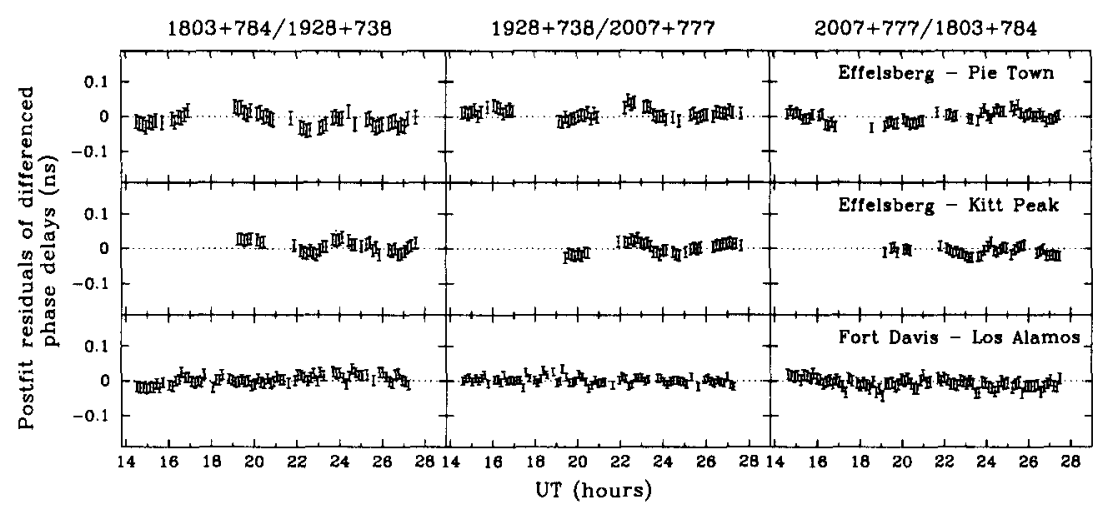

Figure 1. Postfit residuals of the differenced phase-delay observable versus UT for three selected baselines out of the ten used in the astrometric data reduction.

The most difficult contribution to the phase delay to deal with is due to the ionospheric plasma. One way to account for it is through GPS satellite data which allow us to estimate the TEC at the zenith for sites nearby to the observing VLBI stations: Pinyon Flats (CA, US) and Wettzell (Germany). We introduce a longitude (time) correction and an appropriate "slant" factor to estimate the TEC in the line of sight of the observed sources for each VLBI antenna (for details, see Klobuchar 1975). The comparison of this estimation with that resulting from dual frequency group-delay data yields excellent agreement.

We obtain estimates of the separation between the radio sources $1803+784$, $1928+738$, and $2007+777$. In Figure 1 we show the postfit residuals of the differenced phase-delays for some baselines. The corresponding uncertainties for the pairs shown in Figure 1 are 210,150 and $250 \mu$ as, respectively.

The test of sky-closure, defined as the "circular" sum of the separately estimated angular separations of the members of each of the three pairs of radio sources is zero within the error bars. This test provides also a consistency check on the reliability of the individual results.

Acknowledgments. ER acknowledges a Fellowship of the Generalitat Valenciana. The National Radio Astronomy Observatory is a facility of the NSF, operated under a cooperative agreement by Associated Universities, Inc.

\section{References}

Klobuchar, J. A. 1975. Air Force Cambridge Research Laboratories Report No. AFCRL-TR75-0502 (NTIS ADA 018862).

Lara, L. et al. 1996. $A \& A, \mathbf{3 1 4}, 672-678$.

Guirado, J. C. et al. 1995. $A \& A, \mathbf{2 9 3}, 613-625$.

Guirado, J. C. et al. 1995. $A J$, 110, 2586-2596.

Sardón, E., Rius, A., \& Zarraoa, N. 1994. Radio Science, 29, 577-586.

Shapiro, I. I. et al. 1979. $A J, 84,1459-1469$.

Witzel, A. et al. 1988. $A \& A, 206,245-252$. 